\title{
Macroscopic findings in microscopic colitis: Two case reports and a review of literature
}

\begin{abstract}
Microscopic colitis has emerged as an increasingly common cause of chronic and debilitating watery diarrhea in the adult population. Although it was initially assumed to be a histopathologic diagnosis, recent review of literature shows specific endoscopic features that are associated with it. We report two cases of collagenous colitis that had evidence of rectal ulceration on colonoscopy. Both of these cases were seen in Caucasian females in their 60s in association with the use of selective serotonin reuptake inhibitors. This is followed by a review of literature on the subject. Further studies are needed to assess an association of medications with macroscopic findings observed in patients with microscopic colitis.
\end{abstract}

Keywords: microscopic colitis; collagenous colitis; ulceration; collagenous gastritis
Volume 7 Issue 4 - 2017

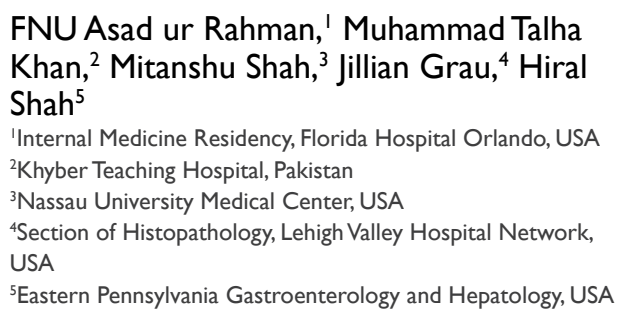

Correspondence: Hiral Shah, Eastern Pennsylvania Gastroenterology and Hepatology, I50 I North Cedar Crest Boulevard \#II0,Allentown, Pennsylvania 18104, USA, Tel 610 821 2828,Email shahshah@gmail.com
Abbreviations: $\mathrm{MC}$, microscopic colitis; COPD, chronic obstructive pulmonary disease; GERD, gastroesophageal reflux disease; EGD, esophago-gastroduo-denoscopy; PPI, proton-pump inhibitors; NSAIDs, non steroidal anti inflammatory drugs; SSRIs, selective serotonin receptor inhibitors

\section{Introduction}

Microscopic colitis (MC) is emerging as an increasingly common cause of chronic and debilitating watery diarrhea in the adult population. The term microscopic colitis was first coined by Park et al in 1980 as an entity causing persistent watery diarrhea. ${ }^{1}$ It was initially characterized by chronic watery diarrhea and normal endoscopic findings with abnormal histopathology on biopsy. ${ }^{2}$ Microscopic colitis comprises two subtypes with lymphocytic colitis and collagenous colitis, based on differing microscopic findings. Lymphocytic colitis is characteristically associated with more than 20 intraepithelial lymphocytes per 100 surface epithelial cells. ${ }^{3}$ Collagenous colitis is characterized by a thick subepithelial collagen band (more than $10 \mu \mathrm{m})$ in the basal membrane. ${ }^{3}$ As awareness and understanding of MC has improved over time, it's documented incidence and prevalence has increased. Although MC was initially conceived to be a histopathologic diagnosis, recent studies have shown specific endoscopic findings that can be associated with MC. ${ }^{4,5}$ We report two cases of biopsy proven collagenous colitis that had macroscopic features on endoscopic examination.

\section{Case I}

A 67 year old caucasian female with past medical history significant for chronic obstructive pulmonary disease (COPD), gastroesophageal reflux disease (GERD) and dyslipidemia was evaluated for persistent watery diarrhea without blood or mucus, for two months. She reported concomitant lower abdominal cramps and tenesmus. She denied any weight loss or fever. She endorsed mild improvement in the frequency of diarrhea after the use of Imodium. Her medication regimen at home included Lansoprazole, Escitalopram, Losartan and Fluticasone/ Salmeterol. Physical examination did not show any features suggestive of malabsorption or systemic illness. Stool cultures were negative for common bacterial and viral infection. Colonoscopic evaluation revealed a deep solitary rectal ulcer, located $30 \mathrm{~cm}$ from the anal verge. Random colonic biopsies were also undertaken from areas of macroscopically normal colonic mucosa. Histopathology from the ulcer showed acute neutrophilic exudate on the background of increased collagen deposition beneath the surface epithelium. Pathology from normal appearing colonic mucosa also showed thickened layer of collagen below the lamina propria consistent with collagenous colitis. Based on these findings, the patient was started on oral budesonide, and Lansoprazole was discontinued. This led to a substantial improvement in symptoms and resolution of diarrhea. Follow up colonoscopy after two months did not show any evidence of mucosal ulceration. Biopsy of the colon revealed improvement in patchy thickening of subepithelial collagen.

\section{Case 2}

A 62 year old caucasian female with a known history of major depressive disorder presented with a two month history of diarrhea. She reported the stool as watery, without any evidence of mucus or blood. She also associated urgency, tenesmus and lower abdominal discomfort with up to fifteen episodes of diarrhea per day. She also reported intermittent epigastric pain and nausea for a similar duration. The patient denied any weight changes, fever, recent travel or use of antibiotics. Her only medication was Venlafaxine. She reported mild epigastric tenderness on examination. Complete blood count and comprehensive metabolic panel did not show any abnormalities. Fecal testing was negative for Clostridium difficile and other infectious agents. In light of epigastric pain, and lower abdominal cramps and diarrhea, esophagogastroduodenoscopy (EGD) and colonoscopy were undertaken. EGD revealed antral gastritis with biopsy findings showing collagenous thickening of the mucosal basement membrane, consistent with collagenous gastritis (Figure 1). Colonoscopic 
examination was significant for a rectal ulcer (Figure 2), but was otherwise unremarkable for any evidence of colitis. Biopsy from the ulcer and the normal appearing colon was consistent with collagenous colitis (Figure 3). Oral budesonide was initiated and venlafaxine was discontinued. This resulted in resolution of diarrhea and abdominal pain. Budesonide was tapered off with no subsequent recurrence of diarrhea. Follow up EGD and colonoscopy showed resolution of gastritis and rectal ulcer (Figure 4).

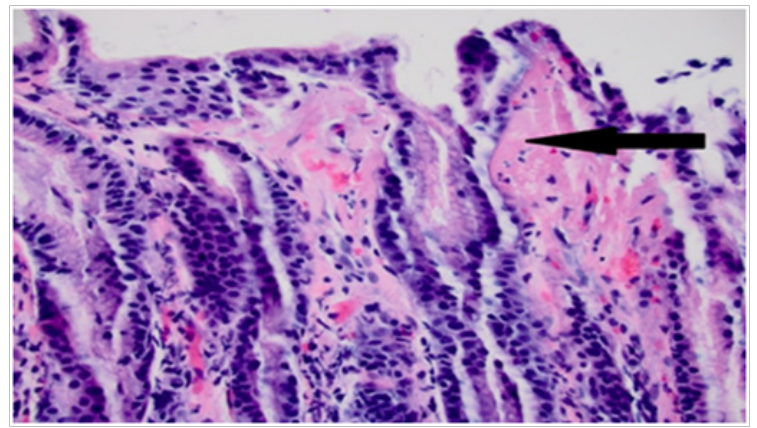

Figure I Gastric antral mucosa demonstrating erosive gastritis with overlapping features of collagenous gastritis.

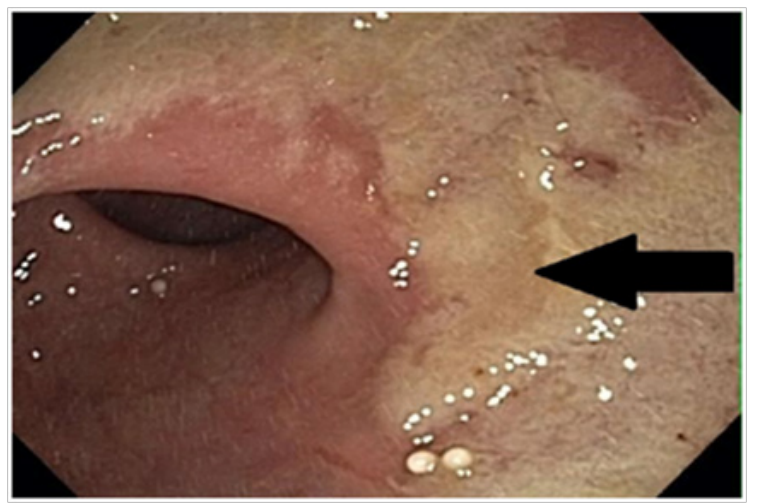

Figure 2 Rectal ulceration observed during colonoscopy.

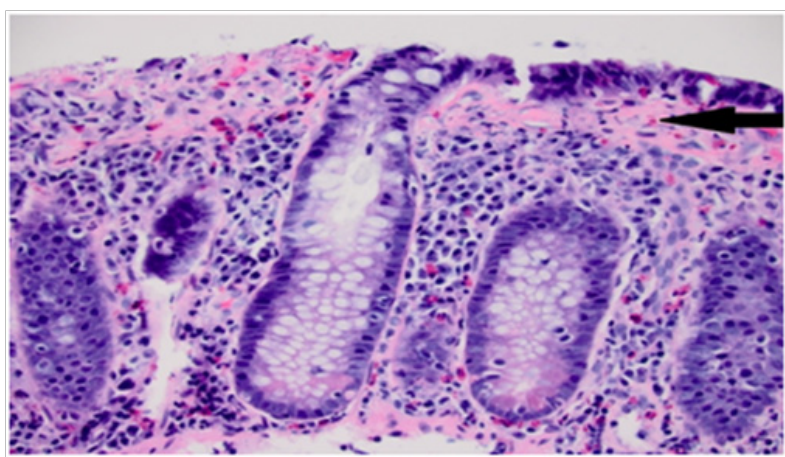

Figure 3 Colonic mucosa with preserved crypt architecture demonstrating plasmacytosis of the superficial lamina propria and thickening of the subepithelial collagen layer to approximately 55 microns.

\section{Discussion}

Microscopic colitis is an important differential diagnosis in patients with chronic diarrhea. A systematic review by Tong et al. ${ }^{6}$ reports an increasing trend in the incidence of MC till the year 2000. The incidence has been stable since then in United States. Overall incidence rate of collagenous colitis (CC) is reported as 4.14 (95\% confidence interval (CI) 2.89-5.40) per 100,000 person-years and
4.85 (95\% CI, 3.45-6.25) for lymphocytic colitis (LC). Female to male incidence rate ratios were 3.05 (95\% CI 2.92-3.19) for CC and $1.92(95 \%$ CI $1.53-2.31)$ for LC (6). It is a disease of the elderly population. The median age at diagnosis for $\mathrm{CC}$ was 64.9 (range, 57.03-72.78) years, similar to LC (median 62.18, range 53.9970.38). ${ }^{6}$ Multiple mechanisms of chronic diarrhea in collagenous colitis have been described:

a. Decreased net absorption of sodium and chloride

b. Active secretion of chloride

c. Reduced water reabsorption secondary to barrier effect of collagen band. ${ }^{7}$ LC and CC may have different pathophysiologic mechanisms at play, it is difficult to distinguish them on a clinical basis, since their presentation is common.

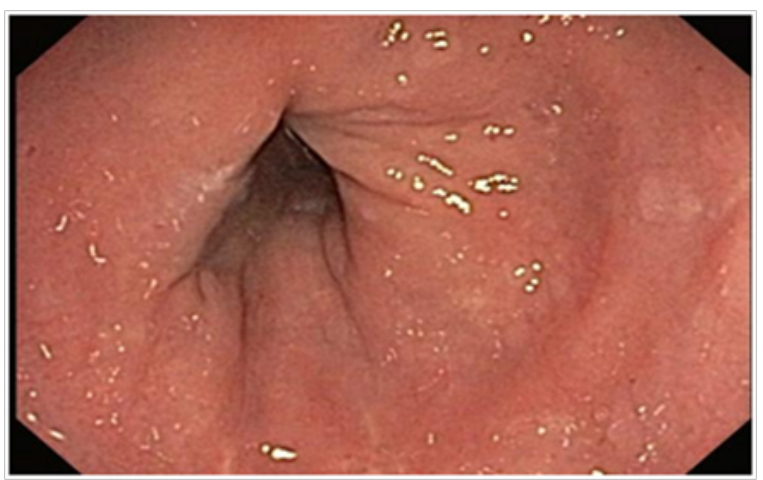

Figure 4 Resolution of rectal ulceration after discontinuation of venlafaxine and treatment with budesonide.

A number of risk factors can predispose patients to develop MC. Several medications have been associated with drug induced microscopic colitis. Proton-pump inhibitors (PPI) appear to have the strongest association. ${ }^{5}$ In a study done by Beaugerie and Pardi, 10 medications including PPIs, Non steroidal anti inflammatory drugs (NSAIDs), and selective serotonin receptor inhibitors (SSRIs) were ranked as high-risk drugs for developing MC. ${ }^{8}$ Smoking appears to be an independent risk factor for development and persistence of MC. ${ }^{9,10}$ Another important consideration is the increased incidence of $\mathrm{MC}$ in patients with other autoimmune diseases. ${ }^{11}$ Associations exist mostly with celiac disease, type 1 diabetes mellitus, autoimmune thyroiditis, or Takayasu's arteritis may be associated with MC. ${ }^{12}$ Despite being termed "microscopic" colitis, there have been a number of cases reporting distinct endoscopic findings in patients with collagenous colitis.

Richieri et al. ${ }^{13}$ first described the presence of multiple linear mucosal lacerations with sharp edges in the right colon of a 43-yearold female, with subepithelial collagen table thickness of $30-40 \mu \mathrm{m}$, in 1993. ${ }^{13}$ Since then, there have been more than sixty reports of distinct colonoscopic findings observed in patients with collagenous colitis. Distinct findings nclude:

a. Pseudomembranes

b. Mucosal vascular abnormalities

c. Mucosal nodularity or textural abnormality

d. Continuum of mucosal breaks and lacerations including cat scratch colon or linear cicatricial lines. ${ }^{4}$

Prevalence of mucosal tears as seen in our patients, are estimated to be around $1 \%$ based on prior reported studies. ${ }^{4}$ However, the true 
number may be higher since not all of these cases are not reported. In addition, practices vary worldwide and up until recently flexible sigmoidoscopy was considered sufficient to diagnose MC. As lesion awareness rises, the incidence of macroscopic findings will increase.

The underlying pathophysiology of mucosal tears has been attributed to thickened abnormal subepithelial collagen that leads to loss of attachment with the epithelial component. This in turn results in stretching of the mucosa over the deeper wall layers, with eventual tearing of the mucosal surface. This sharply demarcated margin of these mucosal defects can help to differentiate these lesions from ischemic colitis. ${ }^{14}$ Most mucosal abnormalities in collagenous colitis are more likely to be found in the right colon since it has a thinner wall and its expansion to a greater diameter during fecal storage and transit, produces greater relative wall tension. ${ }^{15,16}$ These abnormalities can be a risk factor of perforation. ${ }^{17}$ A review by Hussain et al., reports 21 cases of perforation in CC. The majority of these were either colonoscopy associated (15 cases) or barium enema-associated (four cases), while the rest seem to have occurred spontaneously. ${ }^{18}$

In a study of 795 patients, Mellander et al., ${ }^{19}$ found endoscopic abnormalities in $37 \%$ of patients with $\mathrm{CC}$ and $25 \%$ of patients with LC. However, this spectrum of endoscopic abnormalities is seen in other diseases of the colon as well, thus making these findings non specific. Microscopic colitis is diagnosed on the basis of characteristic histologic findings, adequate endoscopic sampling is key. It is recommended to take at least two biopsies per segment, to ensure a high degree of likelihood of detect findings suggestive of $\mathrm{CC}$ or LC. ${ }^{20}$ Key diagnostic components for the diagnosis of MC is defined as subepithelial collagenous band $>10 \mu \mathrm{m}$ in thickness for CC and increase of intraepithelial lymphocytes ( $>20$ per 100 epithelial cells) for LC. ${ }^{21}$

Once MC is diagnosed, the first step in treatment is to identify risk factors such as inciting medications or smoking. In mild cases, discontinuation of medications and use of antidiarrheal medications can lead to resolution. ${ }^{5}$ Data from randomized trials indicates that Budesonide (9mg up to 8 weeks) increased the probability of experiencing clinical remission by $152 \%$ after 6 to 8 months of follow-up (RR, 2.52; 95\% CI, 1.45-4.4). Clinical response was also matched by histologic remission in these studies. ${ }^{22,23}$ The mean time to induce remission in patients with collagenous colitis was found to be 7 to 13 days. ${ }^{23}$ The overall response may be expected for up to $81 \%$ of MC patients. Regarding maintenance of histological response, metaanalysis showed that budesonide $6 \mathrm{mg}$ reduces the risk of histological relapse by $79 \%$ (RR, $0.21 ; 95 \% \mathrm{CI}, 0.08-0.54) .{ }^{24}$ Other medications that have shown some benefit include bismuth salicylate or mesalazine plus cholestyramine, but they have lesser possibility of successful remission, compared to budesonide. ${ }^{25}$ In severe or refractory patients, immunomodulatory medications (TNF alpha inhibitors) have been tried, ${ }^{26}$ however no large scale studies have shown consistent benefit. Surgery can be considered in patients with severe disease, however this remains a treatment of last resort.

\section{Conclusion}

Our unique cases describe macroscopic features in patients with collagenous colitis as well as the correlation with SSRI. Currently there is no data available that links specific endoscopic findings with medication induced microscopic colitis. Our report would help serve as a starting point for further studies that can help answer this question.

\section{Acknowledgments}

None.

\section{Conflicts of interest}

The authors declare no conflicts of interest.

\section{Funding}

None.

\section{References}

1. Read NW, Krejs GJ, Read MG, et al. Chronic diarrhea of unknown origin. Gastroenterology. 1980;78(2):264-271.

2. Zins BJ, Sandborn WJ, Tremaine WJ. Collagenous and lymphocytic colitis:subject review and therapeutic alternatives. Am J Gastroenterol. 1995;90(9):1394-1400.

3. Nyhlin N, Bohr J, Eriksson S, Tysk C. Systematic review: microscopic colitis. Aliment Pharmacol Ther. 2006;23(11):1525-1534.

4. Koulaouzidis A, Saeed AA. Distinct colonoscopy findings of microscopic colitis:not so microscopic after all? World J Gastroenterol. 2011;17(37):4157-4165

5. Okamoto R, Negi M, Tomii S, Eishi Y, et al. Diagnosis and treatment of microscopic colitis. Clin J Gastroenterol. 2016;9(4):169-174.

6. Tong J, Zheng Q, Zhang C, et al. Incidence, prevalence, and temporal trends of microscopic colitis:a systematic review and meta-analysis. Am J Gastroenterol. 2015;110(2):265-276.

7. Burgel N, Bojarski C, Mankertz J, et al. Mechanisms of diarrhea in collagenous colitis. Gastroenterology. 2002;123(2):433-443.

8. Beaugerie L, Pardi DS. Review article:drug-induced microscopic colitis-proposal for a scoring system and review of the literature. Aliment Pharmacol Ther. 2005;22(4):277-284.

9. Roth B, Gustafsson RJ, Jeppsson B, et al. Smoking and alcohol habits in relation to the clinical picture of women with microscopic colitis compared to controls. BMC Womens Health. 2014;14:16.

10. Fernández-Bañares F, de Sousa MR, Salas A, et al. Impact of current smoking on the clinical course of microscopic colitis. Inflamm Bowel Dis. 2013;19(7):1470-1476.

11. Chande N, Driman DK, Reynolds RP. Collagenous colitis and lymphocytic colitis:patient characteristics and clinical presentation. Scand J Gastroenterol. 2005;40(3):343-347.

12. Fernández-Bañares F, de Sousa MR, Salas A, et al. Epidemiological risk factors in microscopic colitis:a prospective case-control study. Inflamm Bowel Dis. 2013;19(2):411-417.

13. Richieri JP, Bonneau HP, Cano N, et al. Collagenous colitis:an unusual endoscopic appearance. Gastrointest Endosc. 1993;39(2):192-194.

14. Nomura E, Kagaya H, Uchimi K, et al. Linear mucosal defects:a characteristic endoscopic finding of lansoprazole-associated collagenous colitis. Endoscopy. 2010;42(Suppl 2):E9-E10.

15. Yarze JC. Finding mucosal tears in collagenous colitis during colonoscopic insufflation. Gut. 2003;52(4):613-614.

16. Milestone AN, Teare JP, Goldin RD. W1498:Linear ulceration in collagenous colitis. a case series and literature review. Gastrointestinal Endoscopy. 2010;71(5):AB343.

17. Woltjen JA. A retrospective analysis of cecal barotrauma caused by colonoscope air flow and pressure. Gastrointest Endosc. 2005;61(1):3745 .

18. Hussain Z, Kelly S, Clarke A, et al. Colonic perforation in collagenous colitis:a systematic review of a rare complication and guidance on management. Surg Endosc. 2010;24(12):2930-2934. 
19. Mellander MR, Ekbom A, Hultcrantz R, et al. Microscopic colitis:a descriptive clinical cohort study of 795 patients with collagenous and lymphocytic colitis. Scand J Gastroenterol. 2016;51(5):556-562.

20. Fernández-Bañares F, Casanova MJ, Arguedas Y, et al. Current concepts on microscopic colitis:evidence-based statements and recommendations of the Spanish Microscopic Colitis Group. Aliment Pharmacol Ther. 2016;43(3):400-426.

21. Münch A, Aust D, Bohr J, et al. Microscopic colitis:current status, present and future challenges. J Crohns Colitis. 2012;6(9):932-945.

22. Pardi DS, Loftus EV, Tremaine WJ, et al. A randomized, double-blind, placebo-controlled trial of budesonide for the treatment of active lymphocytic colitis. Gastroenterology. 2009;136(5):A519-A520.
23. Miehlke S, Heymer P, Bethke B, et al. Budesonide treatment for collagenous colitis:a randomized, doubleblind, placebo-controlled, multicenter trial. Gastroenterology. 2002;123(4):978-984.

24. Nguyen GC, Smalley WE, Vege SS, et al. Clinical Guidelines Committee. American Gastroenterological Association Institute Guideline on the Medical Management of Microscopic Colitis. Gastroenterology. 2016;150(1):242-246

25. Miehlke S, Madisch A, Kupcinskas L, et al. Budesonide is more effective than mesalamine or placebo in short-term treatment of collagenous colitis. Gastroenterology. 2014;146(5):1222-1230.

26. Esteve M, Mahadevan U, Sainz E, et al. Efficacy of anti-TNF therapies in refractory severe microscopic colitis. JCrohns Colitis. 2011;5(6):612618 